\title{
The relationship between Dietary Inflammatory Index and disease severity and inflammatory status: a case-control study of COVID-19 patients
}

Jalal Moludi ${ }^{1,2,3}$, Shaimaa A. Qaisar ${ }^{4}$, Mohammad Alizadeh $^{5 *}$, Hamed Jafari Vayghan ${ }^{6}$, Mohammad Naemi $^{5}$, Akram Rahimi $^{3}$ and Rihaneh Mousavi ${ }^{5}$

${ }^{1}$ Department of Nutritional Sciences, School of Nutritional Sciences and Food Technology, Kermanshah University of Medical Sciences, Kermanshab, Iran

${ }^{2}$ Research Center for Environmental Determinants of Health (RCEDH), Kermanshah University of Medical Sciences,

Kermanshah, Iran

${ }^{3}$ Clinical Research Development Center, Imam Reza Hospital, Kermanshah University of Medical Sciences, Kermanshah, Iran

${ }^{4}$ Chemistry Department, College of Education, University of Garmian, Sulimmania, Iraq

${ }^{5}$ Nutrition Research Center, Faculty of Nutrition, Tabriz University of Medical Sciences, Tabriz, Iran

${ }^{6}$ Department of Nutrition, School of Health, Arak University of Medical Sciences, Arak, Iran

(Submitted 9 March 2021 - Final revision received 1 August 2021 - Accepted 18 August 2021)

Abstract

Numerous studies have revealed strong relationships between COVID-19 and inflammation. However, the imminent link between diet-related inflammation and the COVID-19 risk has not been addressed before. So, we explored the capability of the Energy-Adjusted Dietary Inflammatory Index (E-DII) to predict the inflammatory markers, incidence and severity of COVID-19. We conducted a case-control study consisting of 120 adults; they had been admitted for COVID-19 at hospital during June and July, 2020. The E-DII score was calculated based on the dietary intake, which was evaluated by a 138-item semi-quantitative food frequency questionnaire. Serum levels of inflammatory markers including the Erythrocyte sedimentation rate (ESR), C-reactive protein (CRP) and White blood cells (WBCs) differential were measured. Severity of disease was assessed by chest radiology criteria. Patients with the maximum pro-inflammatory energy adjusted E-DII score had $7 \cdot 26$ times greater odds of developing COVID-19, as compared to those in tertiles 1 (E-DII ${ }_{\mathrm{T} 3} v$. E-DII ${ }_{\mathrm{T} 1}$ : OR $=7 \cdot 26 ; 95 \% \mathrm{CI} 2.64$ to 9.94, $P<0 \cdot 001$ ). Also, a positive association between E-DII and C-reactive protein (CRP) was observed ( $\left.\mathrm{B}_{\mathrm{E}-\mathrm{DII}}=1 \cdot 37,95 \% \mathrm{CI} 0 \cdot 72,2 \cdot 02\right)$, such that with each unit increase in E-E-DII, the CRP levels were increased by 1.37 units. Furthermore, a significant association was found between E-DII and the severity of disease $\left(\mathrm{B}_{\mathrm{E}-\mathrm{DII}}=0.03,95 \% \mathrm{CI} 0.01,0.06 .0 \cdot 024\right)$. Patients consuming a diet with a higher pro-inflammatory potential were at a greater risk of COVID-19 occurrence; also, the severity of disease was elevated with a high score inflammatory diet.

Key words: Dietary Inflammatory Index: Severity: Inflammation: COVID-19

The outbreak of the novel coronavirus disease, also named 'COVID-19', has led to a devastating pandemic, threatening all humans throughout the world. ${ }^{(1)}$ Currently, the number of confirmed cases is rising very quickly globally; it is an immense threat to the public health. ${ }^{(2)}$ Similar to the Middle East respiratory syndrome corona virus (MERS-CoV) and severe acute respiratory syndrome corona virus (SARS-CoV), COVID-19 is characterised by the lower respiratory infection, causing acute respiratory distress syndromes. ${ }^{(3)}$ Typical clinical symptoms of the confirmed patients with COVID-19 include dry cough, fever, fatigue and diarrhoea. ${ }^{(4)}$ Patients who have received intensive care unit (ICU) care, in particular, have many laboratory abnormalities, showing that the proposed COVID-19 might be related to the cellular immune deficiency, coagulation activation and elevated inflammatory factors. ${ }^{(5)}$ However, the immune mechanism potentially orchestrating the acute mortality among COVID-19 patients is still unidentified. There has been much interest in the study of immune systems as an adjustable risk factor for the prevention of lung functional disability and quick mortality. ${ }^{(6,7)}$ Some studies have proposed a link between some nutrient intakes such as vitamin $\mathrm{D}$ and stimulation of the immune system by vitamin $\mathrm{D}_{3}$ therapy. ${ }^{(8,9)}$

The relations between nutritional status, immunity and infectious diseases have been documented; numerous nutrients are

Abbreviations: CRP, reactive protein;; E-DII, Energy-Adjusted Dietary Inflammatory Index;; ESR, erythrocyte sedimentation rate; ICU, intensive care unit.

*Corresponding author: Mohammad Alizadeh, email mdalizadeh@tbzmed.ac.ir 
recognised to play vital roles in the immune function. ${ }^{(10,11)}$ Likewise, it has been postulated that the subclinical immune dysfunction initiated by micronutrient deficiencies may impact the rate of the human COVID-19 disease progression. ${ }^{(12)}$ Aberrant immune responses lead to the long-term pulmonary damage and fibrosis, ultimately leading to acute respiratory distress and increased mortality. ${ }^{(13,14)}$

Like common viral agents (such as norovirus, adenovirus and influenza), COVID-19 has been revealed to cause a more severe disease and worse outcomes in the immunosuppressed subjects. ${ }^{(15)}$ Since the flaw of the immune system is one of the main causal factors leading to some infections due to such viruses, and inflammation is a main causative factor leading to the mortality of COVID-19 subjects, improvement of the immune system and/or anti-inflammatory properties may decrease the incidence and mortality of COVID-19. ${ }^{(5,7)}$ Therefore, a wide range of dietary interventions have been identified to boost the immune response and/or reduce inflammation. ${ }^{(16,17)}$

The Energy-Adjusted Dietary Inflammatory Index (E-DII $\left.{ }^{\circledR}\right)$ is a population-based scoring system established to calculate the inflammatory potential of diets in various populations. ${ }^{(18)} \mathrm{A}$ greater E-DII indicates a higher pro-inflammatory diet, whereas a lower one represents a more anti-inflammatory pattern. ${ }^{(19)}$ The E-DII score has been confirmed by inflammatory markers, such as IL-1 and IL-2, C-reactive protein (CRP) and TNF- $\alpha .{ }^{(20)}$ Hence, evaluating the potential effect of the diet on the inflammation might help to update dietary approaches for the purpose of decreasing the levels of inflammation markers and the risk of COVID-19.

To the best of our knowledge, there has been no study examining the relation between E-DII and COVID-19 risk, and its severity in a COVID-19-infected population. These data are significant because, along with the study of the effects of the nutrient defects on the immune parameters, the impact on clinical outcomes and also, inflammatory markers, if available, should be investigated.

\section{Methods}

This research study was a hospital-based case-control study conducted during June and July 2020. To determine the sample size, the type I error of $\alpha=5 \%$, power $=80 \%$ (type II error of $\beta=20 \%)$ and the groups ratio of 1 was assumed. Based on the study conducted by Abdollahpour et al. ${ }^{(21)}$ in which the proportion of E-DII $>1$ in the control group was estimated at $13.5 \%$, and considering the expected proportion of E-DII $>1$ in the case group equal to $35 \%$ based on the expert opinion, the minimal required sample size was estimated as 60 cases in each groups. Stata software version 13 (StataCorp) was used to determine the sample size. To conduct a retrospective case-control investigation, totally, 120 participants (60 patients with COVID-19 and 60 healthy controls) were included. Patients with positive testing for COVID-19 without any levels of unconsciousness, who had been hospitalised to the Emam Reza Hospital, in Tabriz city, Azerbaijan province, and Golestan Hospital, in Kermanshah city, Kermanshah province, both in Iran, were considered for the case group, from June to July 2020. Patients were requested to contribute to the study after recovery if they wished. In cases they could not answer that question fairly, we asked the close family members of the patients to help the subjects to complete the questionnaires. Patients were considered suitable for registration if they were appropriate for enrolment criteria based on a positive PCR test result; their agreement to keep to the study visit plan and laboratory testing was also obtained. The diagnosis of COVID-19 was done based on clinical evaluation, chest computed tomography and PCR testing, according to the WHO provisional guidance. ${ }^{(22)}$ Further, all patients were categorised into mild, moderate and severe cases based on the results obtained from both clinical examinations, laboratory findings and the chest radiology data. As for controls, 60 patients were enrolled from the same hospitals. They had referred to the outpatient ward for various medical issues (skin diseases, for trauma and injuries, fractures and sprains). Participants in the control group were asymptomatic and negative as tested for COVID-19. Exclusion criteria for both groups were the prior diagnosis of inflammatory diseases such as multiple sclerosis, rheumatoid arthritis, cancer and liver diseases, endocrine disorders, renal or CVD, pregnancy, incomplete answers to questionnaires, dietary under- and/or over-reporting (energy intake outside the range of 3347-17572.8 kJ per d), and those adhering to some special diets including weight loss or a vegetarian diet. To avoid recall biases, the research methodologies were parallel in the two centres. The clinical data were obtained by a standardised questionnaire in the patients' rooms.

Contributors were assessed by a research nurse and/or a physician at the registration visit, following the study visits. The dietitian completed the FFQ survey during the disease. Demographic information, laboratory tests, medical history, chest computed tomography findings and in-hospital mortality were all recorded. Blood sampling and computed tomography examinations were performed within $24 \mathrm{~h}$ of admission. Co-morbidity was established by the patients' self-report of history, physician-documented history or diagnosis during hospitalisation.

This study was approved by the Ethics Board of Kermanshah and Tabriz Universities of Medical Sciences IR.KUMS. REC.1399.444 and IR.TBZMED.REC.1399.225, respectively. All patients signed their written consent after it was explained to them.

\section{Outcomes measurement}

Weight and height were measured upon admission or according to the patients' self-reporting. BMI was computed by dividing the subjects' weight by the square of height.

Baseline questionnaires including demographic information, socio-economic status, physical activity, smoking status, dietary habits, medical history and FFQ were completed in face-to-face interviews by a trained interviewer for all participants. A structured questionnaire was then designed to collect other outcomes such as the length of stay in the hospital and ICU, and unspecific symptoms including fever, dyspnoea and gastrointestinal 
symptoms (vomiting, nausea and diarrhoea); this was done by a physician blinded to the study.

\section{Depression}

The Beck Depression Inventory II (BDI-II), which is a selfassessment questionnaire with 21 items, was applied to measure the presence and severity of depressive symptoms. The total score was calculated, following some cut-off criteria: 0 to 13 , no depression, 14 to 19 , mild depression, 20 to 28 , moderate depression and 29 to 63 , severe depression.

\section{Dietary Inflammatory Index}

E-DII was calculated based on a 138-item semi-quantitative FFQ consisting of a list of foods with standard serving sizes commonly consumed by Iranians. ${ }^{(23,24)}$ Participants were asked to report how often they consumed each of the food items listed as the number of times per $\mathrm{d}$, per week, per month or per year during the previous year. Then the reported frequency for each food item was converted to a daily intake. Portion sizes of the consumed foods were converted to grams using the household measures. The questionnaire had been previously validated for healthy Iranian population.

This interviewer-administered FFQ consisted of 138 food items with typical serving sizes; participants were requested to identify their intake frequency for each food item. Nutrient intake was then measured using the revised Nutritionist IV software (Nutritional Database Manager $4 \cdot 0 \cdot 1$, First Data Bank). FFQ-derived dietary data were applied to compute DII for the participants. Subjects who reported an energy intake outside the range of 3347$17572.8 \mathrm{~kJ} / \mathrm{d}$ were omitted from analysis. We obtained the dietary information of this questionnaire; then the E-DII score was calculated based on the method developed by Shivappa et $_{\text {al }}{ }^{(18)}$ In this method, forty-five food items were introduced, which played a role in the decrease or increase of inflammation. In this study, among these food items and nutrients, we only accessed thirtyone; these included: onion, garlic, coffee, tea, energy, protein, carbohydrates, fibre, vitamins $\mathrm{C}, \mathrm{D}, \mathrm{A}, \mathrm{E}, \mathrm{B}_{6}, \mathrm{~B}_{12}, \mathrm{~B}_{1}, \mathrm{~B}_{2}, \mathrm{~B}_{3}$, folate, $\beta$-carotene, SFA, total fat, MUFA and PUFA, $n$ - 3 and 6 , cholesterol, $\mathrm{Mg}, \mathrm{Fe}$, se and $\mathrm{Zn}$. These food items intakes were linked to the world database mean; their standard deviation was obtained from eleven studies conducted worldwide. ${ }^{(18)} \mathrm{A} Z$-score was determined for each of these mentioned items and participants by subtracting the standard global mean from the participants' dietary intake, as reported; after that, it was divided by standard deviation. We transformed this value to the centred percentile score to minimise the right skewing effect. For this purpose, we multiplied the $Z$-score in 2 and subtracted it by one unit. Finally, all food items specified for the E-DII score were summed, creating the E-DII score. A higher E-DII was associated with the pro-inflammatory diet, while a low one was related to the anti-inflammatory diet.

\section{Biochemical variables}

After fasting for $12 \mathrm{~h}$, blood samples were drawn from the antecubital vein. CRP concentration was assessed via immunoturbidimetry. Erythrocyte sedimentation rate (ESR) was also assayed. ESR was considered high when it was more than 20 $\mathrm{mm}$ in women and 20 in men. The complete blood count test and the leucocytes differential were examined according to standard haematological methods, via Coulter S.

\section{Statistical analysis}

Statistical tests were done via SPSS (version 16.0. Chicago, SPSS Inc.); all $P$-values were according to the two-sided tests. Continuous data with normal or non-normal distribution were described using mean and standard deviation or median (25th, and 75th percentile), respectively. Mann-Whitney $U$ test and independent-samples $t$ test were used to compare continuous variables. Comparison of the case and controls was done in terms of features through the independent-samples $t$ test designed for continuous variables and categorical variables by applying the $\chi^{2}$ tests. E-DII was then analysed as tertiles across the dietary intakes. And $95 \% \mathrm{CI}$ and $\beta$ estimates for some related clinical factors including ESR and CRP were assessed by linear regression. OR and $95 \% \mathrm{CI}$ for the risk of COVID-19 as the outcome were expected via logistic regression models, adjusting only for the confounding factors.

\section{Results}

\section{Participant characteristics}

Of all individuals who joined this study, ten patients in the COVID-19 group and ten in the controls were excluded because of lacking data and diagnosis of additional specific diseases (Fig. 1). Finally, 120 patients (60 cases and 60 controls) were kept for the final analysis.

Table 1 shows the distribution of some features across case and control groups. There were no statically significant differences in terms of age, BMI, sex and medical history among the two groups. E-DII scores in the current study ranged from $2 \cdot 5$ (extreme anti-inflammatory score) to $+4 \cdot 1$ (maximum proinflammatory score). Also, the case group had higher values of E-DII, as compared with the control groups (1.64 v. -0.66 , $P=0 \cdot 001)$. Also, Table 1 compares the data on some inflammatory markers including CRP and ESR. The analysis did confirm significant differences between two groups in terms of CRP and ESR $(P<0 \cdot 001)$. Diabetes was present in $30 \%$ and $21 \%$ of the patients in the case and control groups, respectively $(P=0 \cdot 604)$.

Comparison of the dietary intake in the case and control groups is presented in Table 2. Except for carbohydrates $(P=0.034)$, there were no significant differences in the dietary intake of the two groups, based on the FFQ record $(P>005)$.

Demographic and clinical information, including laboratory parameters, symptoms and signs, and inflammatory markers, are summarised Table 3 . The results of Table 3 were limited to only COVID-19 participants. More than half of the COVID19 patients (69\%) had an elevated body temperature $\geq 37.5^{\circ} \mathrm{C}$. Elevated CRP and ESR were noted in $60 \%$ of the patients (median 9.0, IQR: 3.0-31.0, and median 10, IQR: 4.0-45.5, 


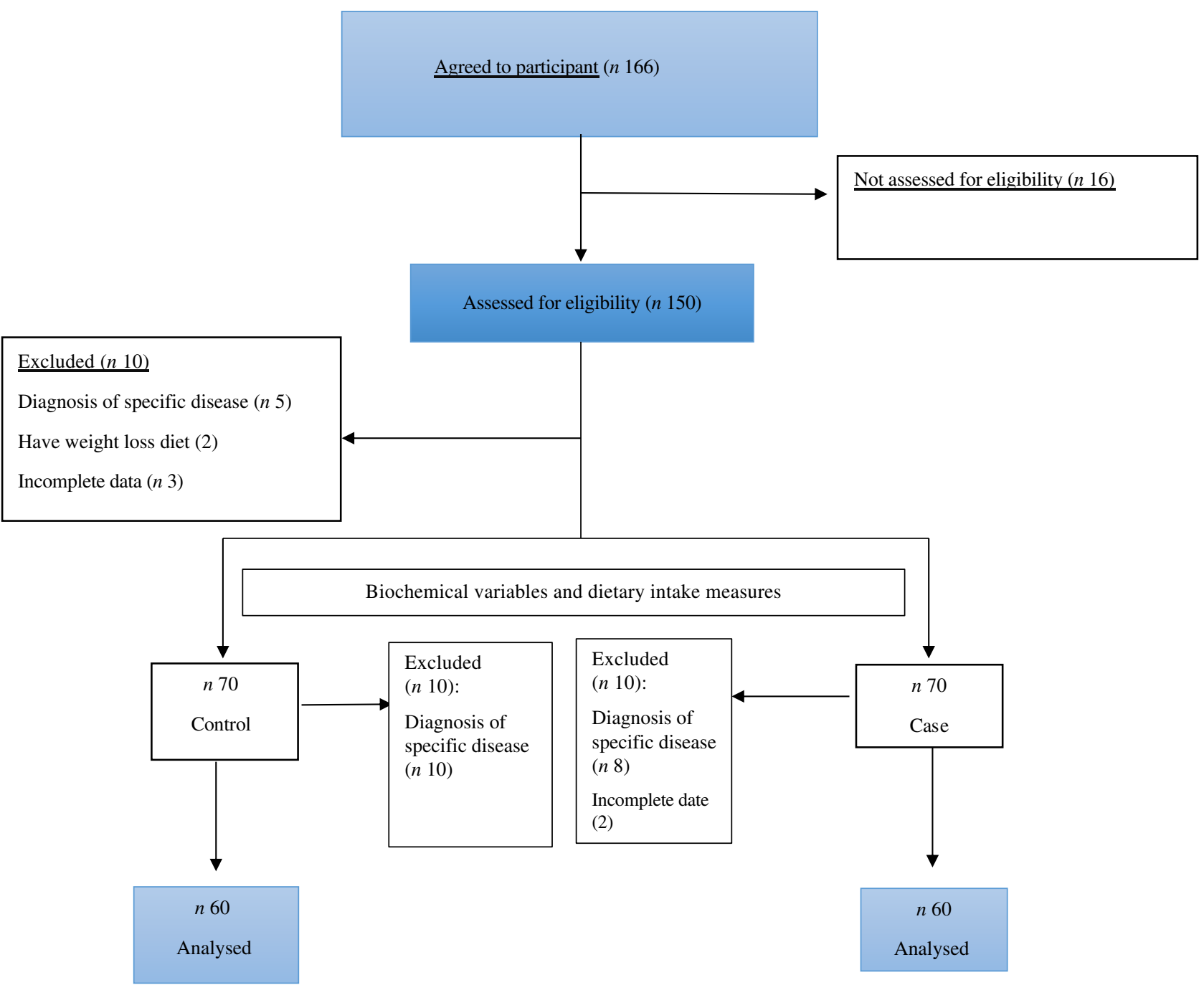

Fig. 1. Flow chart.

respectively). Hb levels were within the normal range. The median duration hospital stay ranged from 2 to $15 \mathrm{~d}$ (median 5.0, IQR: $4 \cdot 4-5 \cdot 4)$. The average length of the stay in the ICU was $2 \cdot 9$ IQR: $0 \cdot 0-5 \cdot 5 \mathrm{~d}$. The most common symptoms were loss of appetite (57\%), GI symptoms (67\%), dyspnoea (73\%) and fever (70\%). One patient (patient 6) remained asymptomatic during the whole course. In our study, there were $35 \%$ mild, $45 \%$ moderate and $20 \%$ severe cases according to the criteria indicated above.

\section{Association of Energy-Adjusted Dietary Inflammatory} Index and risk of COVID-19

OR and $95 \%$ CI for COVID-19 across tertiles of E-DII are presented in Table 4 . In the unadjusted models, subjects with higher E-DII scores were at a higher risk of COVID-19 (E-DII $\mathrm{T}_{3} v$. E-DII T1) (OR: 7.26, $P<0 \cdot 001)$; and this association remained to be significant after adjustment for the confounding factors in the model 3 (OR: 11.86, $P<0 \cdot 001$ ). After multivariable adjustment, subjects with E-DII $>2$ were 11.86 times more likely to have COVID-19, as compared with those with E-DII $\leq-0 \cdot 8$.

\section{Association of Energy-Adjusted Dietary Inflammatory Index with inflammatory and clinical markers in the patients with COVID-19}

As the controls were not admitted in ICU, the data in Table 5 were limited to only COVID-19 participants. E-DII score was positively associated with $\mathrm{CRP}(\mathrm{B}=1.40, P<0.001)$, ESR $(\mathrm{B}=1 \cdot 02, P=0 \cdot 018)$ and $\mathrm{BDI}(\mathrm{B}=1 \cdot 10, P=0 \cdot 025)$, after adjusting for all confounders-; this means that for each unit rise in E-DII, the CRP levels were increased by 1.40 units. However, no significant trend across tertiles of E-DII was observed for hospital and ICU stay (Table 5). Furthermore, a significant association was found between E-DII and the severity of disease (B=0.03, $P=0.024)$.

\section{Discussion}

Increased inflammatory markers including ESR and CRP levels are typically associated with the severity of the COVID-19 disease. Cytokine storms and a series of immune responses induced by COVID-19 have also been observed. The current study was designed to assess the association between the inflammatory 
Table 1. Characteristics of study subjects*

(Mean values and standard deviations; median values and percentiles)

\begin{tabular}{|c|c|c|c|c|c|c|}
\hline \multirow[b]{2}{*}{ Variable } & & \multicolumn{2}{|c|}{ Case $(n 60)$} & \multicolumn{2}{|c|}{ Control $(n 60)$} & \multirow[b]{2}{*}{$P$} \\
\hline & & Mean & SD & Mean & SD & \\
\hline Age (years) $†$ & & $59 \cdot 3$ & 11.8 & 56.03 & 8.8 & 0.107 \\
\hline Weight $(\mathrm{kg}) \dagger$ & & 77.4 & 14.1 & 74.2 & 14.8 & 0.227 \\
\hline BMI $\left(\mathrm{kg} / \mathrm{m}^{2}\right) \dagger$ & & $27 \cdot 3$ & 4.8 & $26 \cdot 51$ & 5.8 & 0.310 \\
\hline E-DII† & & 1.64 & 0.66 & -0.66 & 0.4 & 0.001 \\
\hline \multicolumn{7}{|l|}{$\operatorname{CRP}(\mathrm{mg} / \mathrm{l}) \ddagger$} \\
\hline Median & & $9 \cdot 0$ & & 2.85 & & $<0.001$ \\
\hline Range & & $3.0-31 \cdot c$ & & $1 \cdot 3-4.45$ & & \\
\hline \multicolumn{7}{|l|}{$\operatorname{ESR}(\mathrm{mm} / \mathrm{h}) \ddagger$} \\
\hline Median & & $10 \cdot 0$ & & $5 \cdot 0$ & & $<0.001$ \\
\hline Range & & $4.0-45.5$ & & $2 \cdot 5-13.5$ & & \\
\hline $\mathrm{BDI}+$ & & $20 \cdot 1$ & 9 & $14 \cdot 1$ & 4 & $<0.001$ \\
\hline Death & & 5 & 8.03 & 0 & 0 & $<0.001$ \\
\hline \multirow[t]{2}{*}{ Sex } & Male & 43 & 70 & 42 & 70 & 0.555 \\
\hline & Female & 18 & 30 & 18 & 30 & \\
\hline \multirow[t]{3}{*}{ Activity } & Low & 37 & $60 \cdot 0$ & 40 & 66 & 0.156 \\
\hline & Moderate & 20 & $35 \cdot 0$ & 18 & 30 & \\
\hline & High & 3 & $5 \cdot 0$ & 2 & 4 & \\
\hline \multirow{2}{*}{ Smoking } & Yes & 18 & 30.0 & 10 & $17 \cdot 0$ & 0.523 \\
\hline & No & 43 & $70 \cdot 0$ & 50 & 83.0 & \\
\hline \multirow[t]{2}{*}{ Diabetes } & Yes & 18 & 30 & 13 & 21 & 0.604 \\
\hline & No & 42 & 70 & 47 & 78 & \\
\hline \multirow[t]{2}{*}{ Hypertension } & Yes & 29 & 48 & 21 & 34 & 0.203 \\
\hline & No & 31 & 52 & 39 & 66 & \\
\hline
\end{tabular}

E-DII, Energy-Adjusted Dietary Inflammatory Index, CRP, C-reactive protein; ESR, erythrocyte sedimentation rate; BDI, Beck Depression Inventory.

* Values are expressed as frequency (\%) and $P$-value based on $\chi^{2}$ test.

† Values are expressed as mean (SD) and $P$-value based on Independent-samples $t$ test.

$\mp$ Values are expressed as median (percentile 25 to 75 ) and $P$-value based on Mann-Whitney $U$ test.

Table 2. Comparison of dietary intake in case and control groups*

(Mean values and standard deviations; $95 \%$ confidence intervals)

\begin{tabular}{|c|c|c|c|c|c|c|c|}
\hline \multirow[b]{2}{*}{ Variable* } & \multicolumn{2}{|c|}{ Case $(n 60)$} & \multicolumn{2}{|c|}{ Control $(n 60)$} & \multirow[b]{2}{*}{ MD } & \multirow[b]{2}{*}{$95 \% \mathrm{Cl}$} & \multirow[b]{2}{*}{$P$} \\
\hline & Mean & SD & Mean & SD & & & \\
\hline Energy (kJ) & 8602 & 857 & 8196 & 820 & $-406 \cdot 12$ & $-16 \cdot 38,836 \cdot 78$ & 0.055 \\
\hline Carbohydrates $(\mathrm{g} / \mathrm{d})$ & $304 \cdot 20$ & 65 & $283 \cdot 10$ & 59 & -21.58 & $1.59,-42.78$ & 0.034 \\
\hline Proteins $(g / d)$ & $68 \cdot 20$ & 0.12 & $65 \cdot 20$ & 11 & -3.30 & $-1 \cdot 2,7 \cdot 2$ & 0.134 \\
\hline Fat $(g / d)$ & $70 \cdot 10$ & 16 & $68 \cdot 20$ & 13 & -1.48 & $-3 \cdot 90,6 \cdot 78$ & 0.586 \\
\hline $\operatorname{SFA}(g / d)$ & 28.80 & 7 & $26 \cdot 20$ & 6 & -1.65 & $-6 \cdot 1,4 \cdot 2$ & 0.672 \\
\hline Zinc $(\mathrm{mg} / \mathrm{d})$ & 9.94 & 3.4 & 9.98 & 1.3 & -0.04 & $-1 \cdot 1,0 \cdot 78$ & 0.884 \\
\hline
\end{tabular}

MD: Mean difference.

*Values are expressed as mean (SD) and $P$-value based on Independent-samples $t$ test.

potential of the diet as measured by the E-DII score and the biomarker of inflammation, incidence and severity of COVID-19; we found that the patients with a greater E-DII (i.e. representing a pro-inflammatory diet) were at the amplified odds of having COVID-19 and its severity, thus showing that the intake of a more pro-inflammatory diet was related to the increased risk of COVID-19. It was also revealed that for each one-unit rise in the E-DII score, there was a subsequent increase of CRP and ESR levels, which was by 1.40 and 1.02 units, respectively. Furthermore, compared with the patients in tertiles 1, those in tertiles 3 were 11.86 times more likely to have COVID-19. This may support the hypothesis that the low E-DII score is associated with a lesser amount of severe COVID-19 (as demonstrated for CRP, ESR, etc.)
A number of studies have already recognised that the hyper inflammatory syndrome, triggered by COVID-19, is one of the main causes of disease severity in the infected subjects. ${ }^{(5)}$ Among various factors promoting the setting of an inflammatory milieu in the background of COVID-19, dietary intakes may play a vital role, as they contribute to the onset of various chronic diseases. ${ }^{(10,11)}$ Although there are numerous studies in the literature that have addressed the interactions between nutrients and inflammation, ${ }^{(9,17)}$ no study had yet specified the association between the DII score and the COVID-19 risk. In our sample, the association of E-DII with increased COVID-19 was observed, which was consistent with the previous results obtained for DII and many clinical settings. ${ }^{(19,20)}$ Effect sizes for the diseases risk have generally been in the range of nearly OR 1.5 to 5 , which is 
Table 3. Summary of clinical information of patients with COVID-19 (Numbers and percentages, $n 60$ )

\begin{tabular}{|c|c|c|}
\hline Symptoms and signs & $n$ & $\%$ \\
\hline Body temperature $\geq 37.5^{\circ} \mathrm{C}$ & 39 & $69 \%$ \\
\hline Loss of appetite & 34 & $57 \%$ \\
\hline Gl symptoms & 40 & $67 \%$ \\
\hline Dyspnoea & 44 & $73 \%$ \\
\hline \multicolumn{3}{|l|}{ Severity classification } \\
\hline Mild & 21 & $35 \%$ \\
\hline Moderate & 27 & $45 \%$ \\
\hline Severe & 12 & $20 \%$ \\
\hline \multicolumn{3}{|l|}{ Day with fever above $38^{\circ} \mathrm{C}$} \\
\hline Median & $2 \cdot 0$ & \\
\hline IQR & $0 \cdot 0-3 \cdot 0$ & \\
\hline \multicolumn{3}{|l|}{ Day of hospital stay } \\
\hline Median & $9 \cdot 0$ & \\
\hline IQR & $6 \cdot 0-11 \cdot 0$ & \\
\hline \multicolumn{3}{|l|}{ Day of ICU stay } \\
\hline Median & $2 \cdot 9$ & \\
\hline IQR & $0.0-5.5$ & \\
\hline \multicolumn{3}{|l|}{$\mathrm{Hb},(\mathrm{g} / \mathrm{dl})$} \\
\hline Median & $12 \cdot 0$ & \\
\hline IQR & $11 \cdot 0-12 \cdot 5$ & \\
\hline \multicolumn{3}{|l|}{ Platelet count $\left(\times 10^{9} / \mathrm{I}\right)$} \\
\hline Median & $188 \cdot 0$ & \\
\hline IQR & $167 \cdot 0-199 \cdot 0$ & \\
\hline \multicolumn{3}{|l|}{ Leucocytes count $\left(\times 10^{3} / l\right)$} \\
\hline Median & $6 \cdot 6$ & \\
\hline IQR & $5 \cdot 1-9 \cdot 4$ & \\
\hline Neutrophil (\%) & $71 \cdot 6$ & \\
\hline Lymphocyte (\%) & $17 \cdot 7$ & \\
\hline Creatinine (mg/dl) & 2.9 & \\
\hline Median & $0.0-5.5$ & \\
\hline \multicolumn{3}{|l|}{ IQR } \\
\hline \multicolumn{3}{|l|}{ CRP (mg/l) } \\
\hline Median & $9 \cdot 0$ & \\
\hline IQR & $3 \cdot 0-31 \cdot 0$ & \\
\hline \multicolumn{3}{|l|}{$\mathrm{ESR}(\mathrm{mm} / \mathrm{h})$} \\
\hline Median & $10 \cdot 0$ & \\
\hline IQR & $4 \cdot 0-45 \cdot 5$ & \\
\hline
\end{tabular}

ICU, intensive care unit; CRP, C-Reactive protein; ESR, erythrocyte sedimentation rate.

comparable to our results. ${ }^{(19,25)}$ By comparing these with the results obtained from this study, we hope to define the association between E-DII and COVID-19. The E-DII scores in the current population were comparable to those of the earlier studies $^{(18,26-28)}$. The development of the pro-inflammatory condition could be well correlated with COVID-19; this, in turn, could increase the nitrogen species and reactive oxygen levels, thus playing a vital role in the aetiology and severity of the COVID-19 disease. ${ }^{(7,29)}$ In such a way, the DII score of the diet could be a robust modulator or stimulator of chronic inflammation. ${ }^{(18)}$ Many publications have confirmed that having a healthy diet, which includes high vegetables and fruits, is associated with the lower levels of inflammatory factors. ${ }^{(19,20)}$ Additionally, a particular diet may change the gut microbiota, thus increasing the unsuitable immune function (e.g. modification of the T-helper cell, T-regulatory cell and type 17 and its balance), thus activating the systemic autoimmune diseases. ${ }^{(30)}$ Another plausible explanation of DII and COVID-19 association is that diet can change the expression of different immunity-related genes using epigenetic modification, thus leading to increasing the severity of COVID-19. ${ }^{(31,32)}$ Furthermore, specific dietary factors, such as $\alpha$-linolenic acid and micronutrients with the antioxidant capacity (vitamins E, A and C; Zn, Se), which are included in the DII score, ${ }^{(33)}$ can modulate inflammation. ${ }^{(34)}$

The study also revealed a positive association between following a pro-inflammatory dietary pattern (increase in the EDII score) and the inflammatory markers. There was a strong association between the inflammatory biomarkers used to compute the DII score (CRP, IL- $6, \mathrm{IL}-1 \beta$ and TNF- $\alpha)^{(35)}$ and the severity of COVID-19. In other words, the increased serum levels of several inflammatory cytokines have been associated with disease severity and death ${ }^{(36-38)}$. Uncontrolled inflammatory cell infiltration via the excessive secretion of reactive oxygen species and proteases can itself mediate the damage in the lung, which is the primary cause of death in the COVID-19 disease. ${ }^{(7)}$ Specially, the higher levels of CRP were associated with the lung function. ${ }^{(39)}$ On the other hand, the association between DII and lung function has been reported in a previous study, where DII score and IL- 6 were found to be the negative predictors of the pulmonary function, as indexed by FEV1 (forced expiratory volume in 1s). ${ }^{(25)}$ This inflammation could cause micro-thrombotic immunopathology, leading to pulmonary stress and contributing to mortality. ${ }^{(40)}$ In addition, the massive release of cytokines by the immune system in response to the viral infections can lead to multi-organ failure, especially in the renal, cardiac and hepatic systems. ${ }^{(41)}$ So, controlling the inflammatory response through a low inflammatory diet may be as essential as targeting the virus. Given that the morbidity and mortality seen in COVID-19 is associated with extreme inflammation, a low DII score is useful to develop rationale-based therapeutic strategies.

Table 4. OR and $95 \% \mathrm{Cl}$ of the association between E-DII and COVID-19 (Odd ratio and $95 \%$ confidence intervals)

\begin{tabular}{|c|c|c|c|c|c|c|}
\hline \multirow[b]{2}{*}{ Variable } & \multirow[b]{2}{*}{ E-DII tertile $1(<-0.8)$} & \multicolumn{2}{|c|}{ E-DIl tertile $2(-0.8$ to 2.0$)$} & \multicolumn{2}{|c|}{ E-DII tertile $3(>2.0)$} & \multirow[b]{2}{*}{$P_{\text {for trend }}{ }^{*}$} \\
\hline & & OR & $95 \% \mathrm{Cl}$ & OR & $95 \% \mathrm{Cl}$ & \\
\hline \multicolumn{7}{|l|}{ COVID-19 } \\
\hline Model 1† & Ref & 3.41 & $1.31,8.86$ & $7 \cdot 26$ & $2.64,9.94$ & $<0.001$ \\
\hline Model $2 \ddagger$ & Ref & 2.97 & $1.07,8.19$ & $10 \cdot 74$ & $3.41,13.84$ & $<0.001$ \\
\hline Model $3 \S$ & Ref & 3.06 & $1.07,8.72$ & 11.86 & $5 \cdot 38,18.74$ & $<0.001$ \\
\hline
\end{tabular}

E-DII, Energy-Adjusted Dietary Inflammatory Index.

${ }^{*} P_{\text {for trend }}$ based on logistic regression.

† Unadjusted.

$\ddagger$ Adjusted for age, sex

$\S$ Adjusted for model $2+$ BMI, diabetes, blood pressure. 
Table 5. Association of E-DII with inflammatory and clinical markers in patient with COVID-19 ( $n$ 60)†

\begin{tabular}{|c|c|c|c|c|c|c|c|c|c|}
\hline \multirow[b]{2}{*}{ Variable } & \multicolumn{3}{|c|}{ Model 1‡ } & \multicolumn{3}{|c|}{ Model $2 \S$} & \multicolumn{3}{|c|}{ Model 3II } \\
\hline & B & $95 \% \mathrm{Cl}$ & $P^{\star}$ & B & $95 \% \mathrm{Cl}$ & $P^{\star}$ & B & $95 \% \mathrm{Cl}$ & $P^{\star}$ \\
\hline CRP (mg/dl) & $1 \cdot 37$ & $0.72,2.02$ & $<0.001$ & 1.38 & $0.69,2.08$ & $<0.001$ & 1.40 & $0 \cdot 61,2 \cdot 22$ & $<0.001$ \\
\hline $\mathrm{ESR}(\mathrm{mm} / \mathrm{h})$ & 1.41 & $0.52,2 \cdot 30$ & 0.002 & 1.25 & $0 \cdot 30,2 \cdot 21$ & 0.011 & 1.02 & $0.19,1.98$ & 0.018 \\
\hline Hospital time $(\mathrm{d})$ & 0.17 & $-0.04,0.39$ & 0.112 & 0.13 & $-0.10,0.05$ & 0.264 & 0.13 & $-0.05,0.31$ & 0.155 \\
\hline ICU time $(d)$ & 0.006 & $-0.14,0.15$ & 0.931 & -0.02 & $-0.18,0.14$ & 0.784 & -0.06 & $-0.20,0.12$ & 0.562 \\
\hline Severity & 0.04 & $0.01,0.07$ & 0.005 & 0.03 & $0.01,0.06$ & 0.020 & 0.03 & $0.01,0.06$ & 0.024 \\
\hline
\end{tabular}

E-DII, Energy-Adjusted Dietary Inflammatory Index; CRP, C-reactive protein; ESR, erythrocyte sedimentation rate; ICU, intensive care unit; BDI: Beck Depression Inventory.

${ }^{*} P$ based on linear regression.

† Analysis limited to case group.

‡ Unadjusted.

$\S$ Adjusted for age and sex.

Il Adjusted for Model $1+$ BMl, diabetes and blood pressure.

ๆ Severity of disease were assessed by chest radiology criteria.

Previous research has also revealed an inverse association between healthy diets and markers of inflammation, as well as a direct association with Western dietary patterns. ${ }^{(20,42)}$ Specifically, a lower CRP concentration has been associated with a higher intake of vegetables and fruits, although it is not yet known which degree of inflammation could be attributed to diet. ${ }^{(19)}$ While a complete picture of the effect of nutrition on COVID-19 is far from complete, much development has occurred. Indeed, diet appears to exert a beneficial or detrimental effect via inflammation. Currently, evidence suggests that the usage of DII has numerous unique benefits over other dietary measures, as specially calculated, thus showing the inflammatory potential of diet. ${ }^{(28)}$ Several studies have also revealed that a higher DII score is associated with the elevated levels of inflammatory markers and many diseases including cancer and CVD. ${ }^{(26,27)}$ We showed that the severity of COVID-19 could be partly explained by the high DII score. Chronic inflammation is commonly considered as the explanation for the direct association between DII and COVID-19, which may be via the effect of a pro-inflammatory diet on the systemic inflammation.

Dietary components with a mainly high anti-inflammatory capacity include vitamin E, vitamin C and carotenoids, as indexed by low DII scores, which can interact with transcription factors such as NF- $\mathrm{KB}$, which are related to anti-inflammatory effects. ${ }^{(19,43)}$ These properties are even more serious in view of the exaggerated inflammatory and pro-thrombotic setting associated with COVID-19 severe illness. ${ }^{(44)}$ In addition, dietary fibre, fermented by the gut microbiota into SCFA, has also been displayed to produce anti-inflammatory effects. ${ }^{(16)}$ To summarise, according to the low DII score, having high antioxidant, anti-inflammatory, and potentially antimicrobial and immunomodulatory properties is an encouraging and reasonably easy method to temper the severity of COVID-19 infection.

Another encouraging finding was that a high DII score (i.e. individuals who had the maximum pro-inflammatory diets) could be associated with the severity of COVID-19 (CRP level and severity variable). A similar conclusion was proposed by Phan et al., ${ }^{(43)}$ displaying the relationship between the Mediterranean diet and the severity of psoriasis. As shown, this study was the first one reporting the association of DII with the severity of COVID-19, which could be considered a promising aspect of DII. The mechanisms by which DII might affect COVID-19 remain uncertain. One of the important mechanisms for the detected straight relationship of DII with COVID-19 is through the effect of the pro-inflammatory diet on the levels of various inflammatory cytokines, such as CRP, which, in turn, seem to be significant components in the development and severity of COVID-19. ${ }^{(7,41)}$ A diet including high pro-inflammatory properties (e.g. trans-FA, processed meat) and low antiinflammatory items (e.g. fibres, fruits and vegetables), as indexed by high DII, ${ }^{(18)}$ may play an imperative role in the development of COVID-19.

COVID-19 patients are also likely to suffer from stress and depression as the general population. ${ }^{(45)}$ In the present study, a larger statistically significant difference was observed in the mean BDI values $(20.9 v .14 \cdot 1$, respectively, $P<0.001)$ of the COVID-19 patients, as compared with the control group. Our analyses also showed a linear association between E-DII and depression, as measured by self-reported questionnaires. Inflammatory diets represent a target for behavioural interventions to reduce the health impacts of COVID-19. DII may act as a suitable tool to appraise the impacts of diet and dietary interventions among COVID-19 patients. Interventions to reduce the chronic physiological risk among COVID-19 patients should combine several vital lifestyle changes (e.g. healthy diet and proper sleep). Overall, these results suggest that the inflammatory potential of diet, as assessed by E-DII, was the enhancement of the inflammatory and clinical markers in the patients with COVID-19.

A closer look at the association of the DII score with COVID-19, however, revealed a number of gaps and limitations. First, the number of subjects in this study was relatively small; as such, it is hard to draw a firm conclusion. Second, we did not measure some other cytokine levels including IL-1 $\beta$, TNF- $\alpha$ and IL-6. Further, we did not quantity the socio-economic status, which is an important factor in the food intake and consequently, the DII index, as well as those infected with COVID-19, which serves as a potential limitation. Finally, most patients included in this study were still hospitalised at the time of data collection. So, there might have been some information bias and/or prognosis of the patients. Apart from that, this study was the first one inspecting the association between DII scores and COVID-19. 
These results, therefore, propose the significance of promoting dietary intake with a low DII score for the subjects at the risk of COVID-19. Future studies, including intervention trials, are, however, required to explore this association more precisely.

\section{Conclusion}

To conclude, the present study proposed an inverse association between E-DII score (high in the pro-inflammatory diet) and the risk and severity of COVID-19. In addition, these findings provided additional information regarding chronic inflammation in the development and severity of COVID-19. If these results could be repeated in the next research, an adjusted diet would be one of the multidimensional approaches of COVID-19. Overall, our findings indicated that the intake of more antiinflammatory dietary items such as $n$ - 3 fatty acids, $\beta$-carotenes, phytochemicals, and plant-based foods rich in fibre, and the decrease in the consumption of pro-inflammatory food, such as red and processed meat, trans-FA or acids saturated fat, may be an approach to decrease the risk and severity of COVID-19. This work is of major importance for public healthcare planners, since it could test the low inflammatory diet suggestion at the population basis, offering additional, nonpharmacological lines for the prevention and control of COVID-19.

\section{Acknowledgements}

We would like to thank the Nutrition Research Center, Faculty of Nutrition, Tabriz University of Medical Sciences, Tabriz, Iran.

This research was partially supported by Nutrition Research Center, Faculty of Nutrition, Tabriz University of Medical Sciences, Tabriz, Iran (grant number 65508). Funding organization or sponsor no role in the design and conduct of the study; collection, management, analysis, and interpretation of the data; preparation, review, or approval of the manuscript; and decision to submit the manuscript for publication.

M. A. and M. J. designed the study. R. M. conducted the study and collected the data. H. J. V. performed the statistical analysis. M. J., HJV, MD and RM prepared the manuscript. All the authors have read and approved it.

The authors declare that they have no conflict of interest.

\section{References}

1. Rothan HA \& Byrareddy SN (2020) The epidemiology and pathogenesis of coronavirus disease (COVID-19) outbreak. J Autoimmunity 109, 102433.

2. Lipsitch M, Swerdlow DL \& Finelli L (2020) Defining the epidemiology of Covid-19 - studies needed. N Engl J Med $\mathbf{3 8 2}$ 1194-1196.

3. Hosseiny M, Kooraki S, Gholamrezanezhad A, et al. (2020) Radiology perspective of coronavirus disease 2019 (COVID19): lessons from severe acute respiratory syndrome and Middle East respiratory syndrome. Am J Roentgenol 214, 1078-1082.

4. Qian G-Q, Yang NB, Ding F, et al. (2020) Epidemiologic and clinical characteristics of 91 hospitalized patients with COVID-19 in Zhejiang, China: a retrospective, multi-centre case series. QJM: An Int J Med 113, 474-481.
5. Merad M \& Martin JC (2020) Pathological inflammation in patients with COVID-19: a key role for monocytes and macrophages. Nat Rev Immunol 20, 355-362.

6. Kavianpour M, Saleh M, Verdi J (2020) The role of mesenchymal stromal cells in immune modulation of COVID-19: focus on cytokine storm. Stem Cell Res Ther 11, 1-9.

7. Tay MZ, Poh CM, Rénia L, et al. (2020) The trinity of COVID-19: immunity, inflammation and intervention. Nat Rev Immunol 20, 363-374.

8. Rhodes JM, Subramanian S, Laird E, et al. (2020) Perspective: vitamin D deficiency and COVID-19 severity-plausibly linked by latitude, ethnicity, impacts on cytokines, ACE2, and thrombosis (R1). J Intern Med 289, 97-115.

9. Aman F \& Masood S (2020) How nutrition can help to fight against COVID-19 Pandemic. Pak J Med Sci 36, S121.

10. Terán G (2019) Relation between nutritional status, the outcome of infection and immune responses. https://openarchi ve.ki.se/xmlui/handle/10616/46790

11. Keusch GT (2003) The history of nutrition: malnutrition, infection and immunity. J Nutr 133, 336S-340S.

12. Zabetakis I, Lordan R, Norton C, et al. (2020) COVID-19: the inflammation link and the role of nutrition in potential mitigation. Nutrients 12, 1466.

13. Shi Y, Wang Y, Shao C, et al. (2020) COVID-19 infection: the perspectives on immune responses. Cell Death Differ 27, $1451-1454$.

14. Ong EZ, Chan YF, Leong WY, et al. (2020) A dynamic immune response shapes COVID-19 progression. Cell Host Microbe $\mathbf{2 7}$, 879-882.

15. D'Antiga L (2020) Coronaviruses and immune suppressed patients: the facts during the third epidemic. Liver Transplant 26, 832-834.

16. Iddir M, Brito A, Dingeo G, et al. (2020) Strengthening the immune system and reducing inflammation and oxidative stress through diet and nutrition: considerations during the COVID-19 Crisis. Nutrients 12, 1562

17. Lange KW \& Nakamura Y (2020) Food bioactives, micronutrients, immune function and COVID-19. J Food Bioactives 10, $1-8$.

18. Shivappa N, Steck SE, Hurley TG, et al. (2014) Designing and developing a literature-derived, population-based dietary inflammatory index. Public Health Nutr 17, 1689-1696.

19. Wirth MD, Hébert JR, Shivappa N, et al. (2016) Anti-inflammatory Dietary Inflammatory Index scores are associated with healthier scores on other dietary indices. Nutr Res 36, 214-219.

20. Shivappa N, Steck SE, Hurley TG, et al. (2014) A populationbased dietary inflammatory index predicts levels of C-reactive protein in the Seasonal Variation of Blood Cholesterol Study (SEASONS). Public Health Nutr 17, 1825-1833.

21. Abdollahpour I, Jakimovski D, Shivappa N, et al. (2020) Dietary inflammatory index and risk of multiple sclerosis: findings from a large population-based incident case-control study. Clin Nutr 39, 3402-3407.

22. Lechien JR, Chiesa-Estomba CM, Place S, et al. (2020) Clinical and epidemiological characteristics of 1,420 European patients with mild-to-moderate coronavirus disease 2019. J Intern Med 288, 335-344.

23. Moradi S, Pasdar Y, Hamzeh B, et al. (2018) Comparison of 3 nutritional questionnaires to determine energy intake accuracy in Iranian adults. Clin Nutr Res 7, 213-222.

24. Mirmiran P, Esfahani FH, Mehrabi Y, et al. (2010) Reliability and relative validity of an FFQ for nutrients in the Tehran lipid and glucose study. Public Health Nutr 13, 654-662.

25. Basu A, Devaraj S \& Jialal I (2006) Dietary factors that promote or retard inflammation. Arterioscler Thromb Vasc Biol 26, 995-1001. 
26. Wood LG, Shivappa N, Berthon BS, et al. (2015) Dietary inflammatory index is related to asthma risk, lung function and systemic inflammation in asthma. Clin Exp Allergy 45, 177-183.

27. Deng FE, Shivappa N, Tang Y, et al. (2017) Association between diet-related inflammation, all-cause, all-cancer, and cardiovascular disease mortality, with special focus on prediabetics: findings from NHANES III. Eur J Nutr 56, 1085-1093.

28. Giugliano D, Ceriello A \& Esposito K (2006) The effects of diet on inflammation: emphasis on the metabolic syndrome. $J \mathrm{Am}$ Coll Cardiol 48, 677-685.

29. Ramallal R, Toledo E, Martinez-Gonzalez MA, et al. (2015) Dietary inflammatory index and incidence of cardiovascular disease in the SUN cohort. PLoS One 10, e0135221.

30. Miripour ZS, Sarrami-Forooshani R, Sanati H, et al. (2020) Realtime diagnosis of reactive oxygen species (ROS) in fresh sputum by electrochemical tracing; correlation between COVID19 and viral-induced ROS in lung/respiratory epithelium during this pandemic. Biosens Bioelectron 165, 112435.

31. Chow J \& Mazmanian SK (2009) Getting the bugs out of the immune system: do bacterial microbiota 'fix' intestinal $\mathrm{T}$ cell responses? Cell Host Microbe 5, 8-12.

32. Chlamydas S, Papavassiliou AG \& Piperi C (2020) Epigenetic mechanisms regulating COVID-19 infection. Epigenetics 16, 263-270.

33. Holuka C, Merz MP, Fernandes SB, et al. (2020) The COVID-19 Pandemic: does our early life environment, life trajectory and socioeconomic status determine disease susceptibility and severity? Int J Mol Sci 21, 5094.

34. Del Mar Bibiloni M, Maffeis C, Llompart I, et al. (2013) Dietary factors associated with subclinical inflammation among girls. Eur J Clin Nutr 67, 1264-1270.

35. Ruiz-Canela M, Bes-Rastrollo M \& Martínez-González MA (2016) The role of dietary inflammatory index in cardiovascular disease, metabolic syndrome and mortality. Int J Mol Sci 17, 1265.
36. Chen G, Wu DI, Guo W, et al. (2020) Clinical and immunological features of severe and moderate coronavirus disease 2019. J Clin Investig 130, 2620-2629.

37. Yang Y, Shen C, Li J, et al. (2020) Exuberant elevation of IP10. MCP-3 and IL-1ra during SARS-CoV-2 infection is associated with disease severity and fatal outcome. medRxiv 2002, 2020.

38. Gong J, Dong H, Xia QS, et al. (2020) Correlation analysis between disease severity and inflammation-related parameters in patients with COVID-19: a retrospective study. BMC Infect Dis 20, 1-7.

39. Gimeno D, Delclos GL, Ferrie JE, et al. (2011) Association of CRP and IL-6 with lung function in a middle-aged population initially free from self-reported respiratory problems: the Whitehall II study. Eur J Epidemiol 26, 135-144.

40. McGonagle D, O'Donnell JS, Sharif K, et al. (2020) Immune mechanisms of pulmonary intravascular coagulopathy in COVID-19 pneumonia. Lancet Rheumatol 2, e437-e445.

41. Mangalmurti N \& Hunter CA (2020) Cytokine storms: understanding COVID-19. Immunity 53, 19-25.

42. Nettleton JA, Steffen LM, Mayer-Davis EJ, et al. (2006) Dietary patterns are associated with biochemical markers of inflammation and endothelial activation in the Multi-Ethnic Study of Atherosclerosis (MESA). Am J Clin Nutr 83, 1369-1379.

43. Phan C, Touvier M, Kesse-Guyot E, et al. (2018) Association between Mediterranean anti-inflammatory dietary profile and severity of psoriasis: results from the NutriNet-Santé cohort. JAMA Dermatol 154, 1017-1024.

44. Mehta P, McAuley DF, Brown M, et al. (2020) COVID-19: Consider cytokine storm syndromes and immunosuppression. Lancet 395, 1033.

45. Liu D, Baumeister RF, Veilleux JC, et al. (2020) Risk factors associated with mental illness in hospital discharged patients infected with COVID-19 in Wuhan, China. Psychiatry Res 292, 113297. 